\title{
The role of postoperative adjuvant radiotherapy in local recurrence risk in medullary thyroid carcinoma
}

\author{
Aleksandra Kukulska ${ }^{1}{ }^{2}$, Jolanta Krajewska', Zofia Kolosza ${ }^{3}$, Ewa Paliczka-Cieslik', Aleksandra Kropinska1, \\ Agnieszka Pawlaczek¹, Zbigniew Puch ${ }^{1}$, Kornelia Ficek², Teresa Lisik², Dorota Sygula², Zbigniew Wygoda , \\ Jozef Roskosz ${ }^{1}$, Jerzy Wydmanski² and Barbara Jarzab ${ }^{1}$ \\ ${ }^{1}$ Nuclear Medicine and Endocrine Oncology Department, M. Sklodowska-Curie Institute - Oncology Center, Gliwice Branch, Gliwice, Poland \\ ${ }^{2}$ Radiotherapy Department, M. Sklodowska-Curie Institute - Oncology Center, Gliwice Branch, Gliwice, Poland \\ ${ }^{3}$ Department of Epidemiology and Silesia Cancer Registry, M. Sklodowska-Curie Institute - Oncology Center, Gliwice Branch, Gliwice, Poland \\ Correspondence should be addressed to J Krajewska: jolanta.krajewska@io.gliwice.pl
}

\begin{abstract}
The value of postoperative radiotherapy in the treatment of medullary thyroid carcinoma (MTC) has not been unequivocally demonstrated. Therefore our study aimed to answer the question of whether adjuvant radiotherapy showed any impact on the risk of local recurrence and whether there were any differences in response to radiotherapy between hereditary and sporadic MTC.

Methods: A retrospective analysis involved 254 MTC patients, among them 73 patients with a hereditary disease. Two hundred and twenty-four patients, including 43 persons at a high risk of local relapse, underwent only initial surgery; 18 other patients were operated due to MTC recurrences, whereas the remaining 12 patients had cytoreductive procedure or were not amenable for surgery. Radiotherapy was carried out in 132 patients. One hundred and twenty patients underwent adjuvant radiotherapy, among them 102 patients after initial surgery. The median follow-up was 10 years (range 0.5-29 years).

Results: Local recurrence occurred in 107/254 patients, among them in 63 subjects after prior radiotherapy. The frequency of relapse showed significantly increasing trend toward higher MTC stages $(P<0.001)$. More relapses occurred in patients with lymph node metastases present at MTC onset. Adjuvant radiotherapy was associated with a lower risk of nodal recurrence only in high-risk patients, particularly if lymph node metastases were present at MTC diagnosis. The differences between hereditary and sporadic subgroups were not significant.

Conclusions: Adjuvant radiotherapy has a limited importance in MTC treatment. It should be considered in high-risk MTC patients. The presence of RET mutation does not influence the response to radiation.
\end{abstract}

\section{Key Words}

- medullary thyroid carcinoma

- adjuvant radiotherapy

- postoperative radiotherapy

- radiation treatment
Endocrine Connections (2020) 9, 1-8

\section{Introduction}

Medullary thyroid carcinoma (MTC) is a rarely diagnosed malignant neoplasm arising from $\mathrm{C}$ cells and constitutes only $5 \%$ of all thyroid cancers. MTC may occur as a sporadic disease or as part of hereditary multiple endocrine neoplasia (MEN) syndromes $(1,2,3,4,5)$. Surgery, based on total thyroidectomy and central neck lymph node dissection, completed by a modified lateral neck lymphadenectomy, if indicated, is the main treatment method.

Other standard oncological treatment modalities, including chemo and radiotherapy, demonstrate a 
limited efficacy in MTC (6). Radiotherapy may be applied in MTC patients either as an adjuvant treatment or as a palliative approach. Palliative radiotherapy is an effective and accepted method to treat symptoms related to a local recurrence or MTC metastases such as pain or dyspnea, whereas adjuvant radiotherapy is usually carried out after surgery. However, its value in MTC treatment is still discussed (7). So far, there are no sufficient data to precisely evaluate its effectiveness in MTC due to the lack of randomized clinical trials. Our knowledge, related to the efficacy and safety of adjuvant radiotherapy, is mainly based on small studies coming from one center with different outcomes (2). In 1977 Steinfeld et al. reported postoperative radiotherapy as a therapeutic option in MTC (8). Since then, some papers concerning the effectiveness of radiotherapy at preventing MTC recurrence and its impact on survival have been published. However, the vast majority of them did not demonstrate any improvement in the survival of MTC patients $(9,10)$. Nevertheless, radiotherapy may result in a better locoregional control, particularly in patients showing a high risk of local MTC relapse $(11,12,13,14)$.

The revised MTC ATA guidelines, published in 2015, recommend the selective use of adjuvant radiotherapy in patients who demonstrate a higher risk of local relapse, among them in those after non-radical surgical approach, with extrathyroidal extension, massive nodal involvement or in case of airways compression. However, according to the authors of ATA guidelines, the qualification for radiotherapy has to be cautious due to a risk of acute and late complications (6). Among the most common complications observed during radiotherapy and just after the treatment are dermatitis and mucositis in the esophagus, pharynx, and larynx manifested by pain, swallowing disturbances or even sometimes by dyspnea requiring, in some cases, temporary tracheostomy or percutaneous endoscopic gastrostomy (PEG). The late consequences of radiotherapy involve skin hyperpigmentation, telangiectasias, hoarseness, and xerostomia. Serious complications such as tracheal, esophageal, and blood vessel stenosis or spinal cord injury are rare, particularly when dynamic techniques are used $(6,15)$. Currently, in the era of the use of tyrosine kinase inhibitors (TKIs), the risk of tracheal and/or esophageal fistulas as a potential side effect of radiotherapy is of particular relevance as TKIs may also induce such complications. Therefore, qualification for radiotherapy in patients on TKIs has to be very cautious.

Considering adjuvant radiotherapy, a radiation treatment planning assumes that therapeutic dose targets thyroid bed, regional neck and upper mediastinal lymph nodes. The elective radiation doses range from 50 to $54 \mathrm{~Gy}$, whereas in regions showing positive surgical margins, from 60 to $66 \mathrm{~Gy}$. The macroscopic residual tumor requires a radiation dose of $70 \mathrm{~Gy}$, depending on the treatment tolerance $(4,15,16)$. The use of dynamic techniques and proper verification methods of patient position allows for a better protection of healthy tissues and simultaneously for an increase of radiation doses in target volumes $(4,15,16,17,18)$.

Our study aimed to evaluate the role of postoperative adjuvant radiotherapy in local disease control in MTC patients. We tried to resolve the following questions: whether external beam radiotherapy (EBRT) reduces the risk of MTC recurrence in high-risk patients, whether the dose escalation may improve its effectiveness, what was the role of adjuvant radiotherapy in low-risk patients, and whether adjuvant radiotherapy reduced the risk of thyroid bed or nodal recurrence. We also attempted to answer the question of whether there were any possible differences in a response to radiotherapy between hereditary and sporadic MTC.

\section{Materials and methods}

We retrospectively analyzed MTC course in 254 patients, 172 women and 82 men at a mean age of 47 years, median 46 years. The youngest female was 6 years old and the oldest was 82 years old (the mean and median values were 47 years), whereas the ages of the youngest and oldest males were 25 and 75 years, respectively (the mean and median values: 47 and 46 years, respectively; Table 1). Two hundred and forty-two patients underwent total thyroidectomy; 12 patients had cytoreductive surgery or were not operated.

One hundred and thirty-two patients were subjected to postoperative radiotherapy; among them 120 patients underwent radiotherapy to reduce the risk of local relapse in thyroid bed or regional lymph nodes, whereas the remaining 12 patients to reduce MTC symptoms such as dysphagia, dyspnea or pain (palliative treatment). All patients referred to postoperative radiotherapy had total thyroidectomy. In 102 of them radiotherapy followed the primary thyroid surgery, whereas 18 other patients were operated several times prior to radiation treatment due to local recurrences.

All surgical procedures which did not lead to the resection of the whole macroscopic or microscopic tumor were considered as non-radical surgeries. R1 surgery was defined by the presence of positive margins

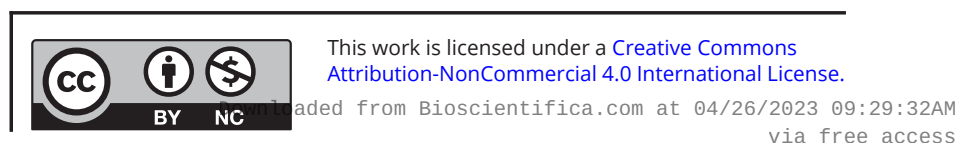


Table 1 Characteristics of the study group.

\begin{tabular}{|c|c|c|c|}
\hline & $\begin{array}{l}\text { Radiotherapy } \\
\text { subroup }\end{array}$ & $\begin{array}{c}\text { No-radiotherapy } \\
\text { subgroup }\end{array}$ & P value \\
\hline \multicolumn{4}{|l|}{ Age (years) } \\
\hline Mean & 47 & 47 & 0.82220 \\
\hline Median & 46 & 48 & \\
\hline Range & $18-77$ & $6-82$ & \\
\hline \multicolumn{4}{|l|}{ Sex } \\
\hline Female & 88 & 84 & 0.711048 \\
\hline Male & 44 & 38 & \\
\hline \multicolumn{4}{|l|}{$\mathrm{T}$ feature } \\
\hline pT1 & 21 & 34 & 0.021094 \\
\hline pT2 & 31 & 49 & 0.004339 \\
\hline pT3 & 27 & 16 & 0.120171 \\
\hline pT4 & 26 & 8 & 0.002176 \\
\hline pTx & 27 & 15 & 0.081147 \\
\hline \multicolumn{4}{|l|}{$\mathrm{N}$ feature } \\
\hline NO & 4 & 38 & $<0.00001$ \\
\hline $\mathrm{Nx}$ & 5 & 22 & 0.000241 \\
\hline N1 & 123 & 62 & $<0.00001$ \\
\hline $\begin{array}{l}\text { Hereditary } \\
\text { disease }\end{array}$ & 29 & 44 & 0.01483 \\
\hline
\end{tabular}

on histopathological examination, without any residual tumor, whereas R2 surgery corresponded to macroscopic residual tumor, confirmed by US, CT or MRI.

Local recurrence was diagnosed when MTC relapse occurred in thyroid bed or regional lymph nodes. Nonradical surgery (R1 or R2), $\mathrm{T} 4$ feature or extranodal extension were considered as risk factors of local recurrence. The group of high risk of local recurrence involved 43 patients. Radiotherapy was carried out in 35 of them.

Among 102 patients in whom radiotherapy was applied after primary thyroid resection 8 had R1 surgery, 3 had R2 surgery, T4 feature was diagnosed in 12 persons and 11 patients had extranodal extension. Ninety-one patients underwent radical surgical procedure (no microor macroscopic residual tumor, negative margins).

Lymph node metastases were diagnosed in 96 patients treated with postoperative radiotherapy, among them in 43/47 patients with T1-T2 feature (91\%). Regarding patients who were not subjected to postoperative radiotherapy, lymph node metastases were present in 63 persons, among them in 37/83 T1-T2 patients (59\%).

According to the histopathological examination, based on TNM classification (6th version, 2002) T1 feature was diagnosed in 16 patients treated with radiotherapy after the first surgery and in 3 patients subjected to radiotherapy after the surgical resection of MTC recurrence. T2 was diagnosed in 23 and 5 patients, T3 in 24 and 1 patient, T4 in 19 and 5 patients, and Tx (tumor diameter not known) in 20 and 4 patients, respectively. Lymph node metastases were present in
96 patients treated with radiotherapy following the first surgery and in 17 patients subjected to radiotherapy after the surgical resection of MTC recurrence (Table 1). Distant metastases were excluded based on chest X-ray, abdominal ultrasound, chest/abdomen $\mathrm{CT}$ and bone scan in all patients qualified for adjuvant radiotherapy.

Twelve patients, who presented gross residual neck tumor, treated with palliative radiotherapy to reduce MTC symptoms had cytoreductive surgery or were not operated. Among them, 10 had lymph node metastases.

In a group of 122 patients in whom radiotherapy was not applied T1, T2, T3, T4, and Tx features were diagnosed in $34,49,16,8$ and 15 persons, respectively. Lymph node metastases were observed in 62 patients. Two patients had R1 surgery. No patient presented MTC symptoms.

The patients underwent radiation treatment between the years 1987 and 2014. Since 1997, radiotherapy planning has been made in a 3-D treatment planning system (CadPlan, VARIAN Medical Systems). Eclipse (VARIAN Medical Systems) has been used for intensity-modulated radiation therapy (IMRT) dose calculations since 2001. Twenty-two patients from the study group were irradiated between 1987 and 1996, 20 patients between 1997 and 2000, whereas the remaining patients between 2001 and 2014 .

Follow-up period started at the first admission of the patients in our institute and ended on the day of database lock. The median and mean follow-up was 10 years (range 0.5-29 years). Two hundred and four patients were monitored longer than 5 years, whereas 16 patients longer than 20 years. In this analysis, PFS (progression-free survival) was considered as a time free of local recurrence. The date of cytological confirmation of MTC recurrence in thyroid bed or lymph nodes was the date of the end of PFS.

DNA analysis for germline mutations of the RET gene was performed in all patients in the Nuclear Medicine and Endocrine Oncology Department, at M. Sklodowska-Curie Institute - Oncology Center in Gliwice, Poland. Genomic DNA was extracted from peripheral blood nucleated cells by the desalting method using Genomic Maxi AX kits (A\&A Biotechnology, Poland). Mutation screening was performed according to a standard algorithm, which assumes the analysis of exons 10, 11, 13, 14, 15 and 16. The results were analyzed with the Applied Biosystems Sequencing Analysis v5.2 software (Applied Biosystems). A hereditary type of the disease was confirmed in 73 patients.

Serum calcitonin concentration was measured during follow-up visits by the use of immunoradiometric determination (IRMA-HCT, Cisbio Bioassays, France).

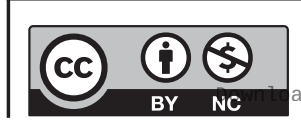

This work is licensed under a Creative Commons Attribution-NonCommercial 4.0 International License. ded from Bioscientifica.com at 04/26/2023 09:29:32AM 
Statistical analysis, carried out using STATISTICA software, version 10 (2011; StatSoft, Inc; USA) and Stata 13.1 for Windows, was based on the following tests: the exact Fisher's test, Mann-Whitney test and Kaplan-Meier estimate. The Cox proportional hazards model was used for the multivariate analysis.

\section{Results}

Local recurrence was observed in 107/254 (42\%) patients from the study group. It was diagnosed more common in patients with higher MTC stages - in 14 (25\%), 30 (37\%), 20 (47\%), 20 (60\%), and 23 (53\%) among pT1, pT2, pT3, pT4 and pTx patients, respectively. The frequency of MTC relapse showed statistically significant, increasing trend toward higher MTC stages $(P<0.001)$. More relapses were also noticed in patients with lymph node metastases at MTC diagnosis: 98/185 (53\%) of all N1 patients.

The further analyses, whose results are given below, were carried out only in a group of 224 patients after initial thyroid surgery. Patients with MTC symptoms, who required palliative radiotherapy, and patients treated due to multiple MTC recurrences were excluded from the analysis.

Local recurrence was diagnosed in 89/224 (40\%) patients, among them in 32 (36\%) patients it was localized in the thyroid bed, whereas in the remaining 57 (64\%) subjects in local lymph nodes. MTC recurrence occurred in 50/102 (49\%) patients treated with adjuvant radiotherapy, among them in 22 patients in thyroid bed and 28 patients in regional lymph nodes. The difference between 'radiotherapy' and 'no-radiotherapy' groups was not significant (Fig. $1 \mathrm{~A}$ and $\mathrm{B}$ ).

Considering a subgroup of 43 high-risk MTC patients, locoregional recurrence was diagnosed in 27 (63\%) patients (Table 2), among them in 13/27 (48\%) subjects in the thyroid bed. Twelve patients (92\%) underwent adjuvant radiotherapy. Nodal recurrence occurred in $14 / 27$ (52\%) patients. Eight of them (57\%) underwent radiotherapy (Fig. 2A and $\mathrm{B}$ ). The risk of thyroid bed recurrence in high-risk MTC patients did not significantly differ between patients with and without adjuvant radiotherapy (relative risk $[\mathrm{RR}]=2.5$; 95\% CI: 0.33-19.38; $P=0.377$ ). While the risk of nodal recurrences in highrisk MTC patients after radiotherapy was significantly lower than in the 'no-radiotherapy' subgroup $(\mathrm{RR}=0.25$; 95\% CI: 0.09-0.74; $P=0.012$ ).

Regarding a subgroup of 159 N1 MTC patients, locoregional recurrence was diagnosed in 82/159 (52\%) patients (Table 2). In 31/82 (38\%) patients it was localized in the thyroid bed. Twenty-two (71\%) of them underwent adjuvant radiotherapy. Nodal recurrence occurred in 51/82 (62\%) subjects, among them 28 (55\%) underwent radiotherapy (Fig. $3 \mathrm{~A}$ and $\mathrm{B}$ ). The risk of thyroid bed recurrence in N1 patients without other high-risk features did not significantly differ between patients who underwent and did not undergo adjuvant radiotherapy ( $\mathrm{RR}=1.14 ; 95 \% \mathrm{CI}: 0.45-2.89 ; \quad P=0.785)$. Similarly, the difference in the risk of nodal recurrence between 'radiotherapy' and 'no-radiotherapy' groups was not significant $(\mathrm{RR}=0.95 ; 95 \% \mathrm{CI}$ : 0.49-1.82; $P=0.869)$.

In a subgroup of 41 high-risk MTC patients with lymph node metastases at MTC diagnosis, locoregional recurrence occurred in 27/41 (66\%) patients, among them in 13 (48\%) subjects in the thyroid bed. Twelve (92\%) of them underwent adjuvant radiotherapy. Nodal recurrence was present in 14 (52\%) patients; 8 of them (57\%) underwent radiotherapy (Fig. 4A and B). The risk of MTC relapse in the thyroid bed did not significantly differ between patients with and without adjuvant radiotherapy $(\mathrm{RR}=2.02 ; 95 \% \mathrm{CI}: 0.26-15.68 ; P=0.502)$. In contrary, the risk of nodal relapse was significantly lower in patients
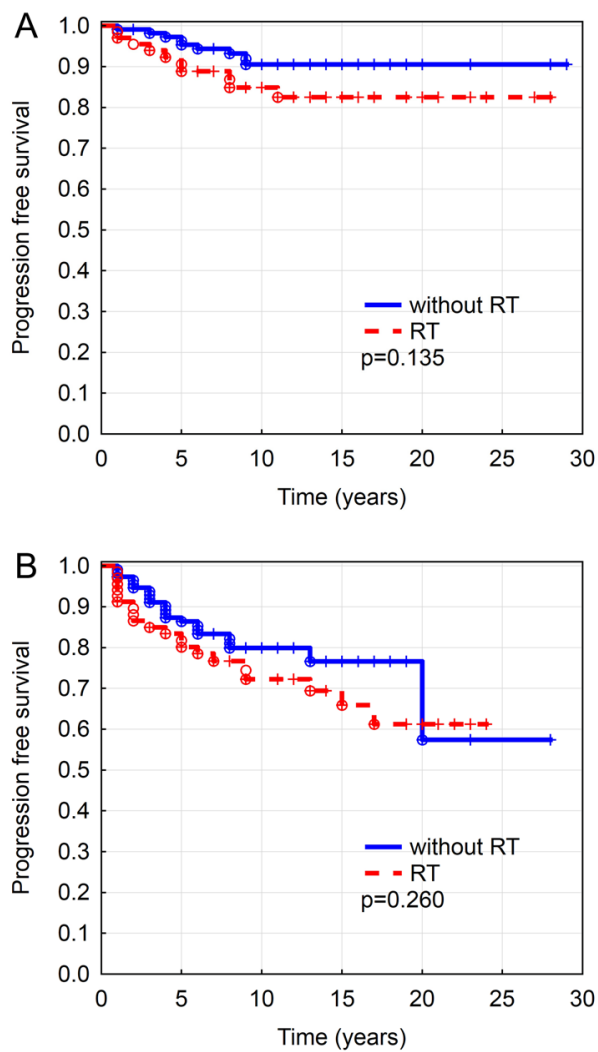

Figure 1

The differences in progression-free survival between patients with (the red line) and without (the blue line) adjuvant radiotherapy following initial surgical procedure. (A) MTC recurrence in thyroid bed. (B) MTC recurrence in lymph nodes. 
Table 2 MTC recurrences with reference to risk group and radiotherapy.

\begin{tabular}{|c|c|c|c|}
\hline & The whole group & Radiotherapy & No radiotherapy \\
\hline High-risk patients & 43 & 34 & 9 \\
\hline N1 patients & 159 & 96 & 63 \\
\hline Group T1-T3N0 & 40 & 3 & 37 \\
\hline
\end{tabular}

\begin{tabular}{l} 
MTC recurrences in \\
radiotherapy group \\
\hline 20 \\
50 \\
0
\end{tabular}

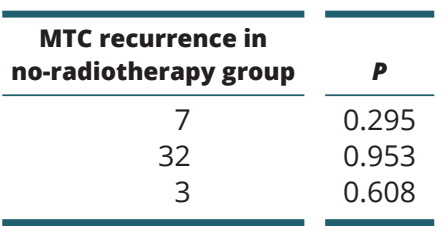

after adjuvant radiotherapy than in subjects without radiotherapy ( $\mathrm{RR}=0.21 ; 95 \% \mathrm{CI}$ : $0.07-0.61 ; P=0.004)$.

The differences in the risk of local relapse in low-risk MTC patients with and without adjuvant radiotherapy were not statistically significant either with reference to thyroid bed ( $\mathrm{RR}=1.97 ; 95 \% \mathrm{CI}$ : $0.80-4.94 ; P=0.141$ ) or with reference to nodal relapses $(\mathrm{RR}=1.41 ; 95 \% \mathrm{CI}$ : 0.77-2.58; $P=0.267$; Table 2).

In a hereditary subgroup (73 patients), local relapse was diagnosed in 25 (34\%) subjects, among them in 13 (52\%) patients, who underwent radiotherapy. In a subgroup of 181 patients with sporadic MTC, the relapse was observed in $82(45 \%)$ subjects. Fifty persons (61\%) with recurrent
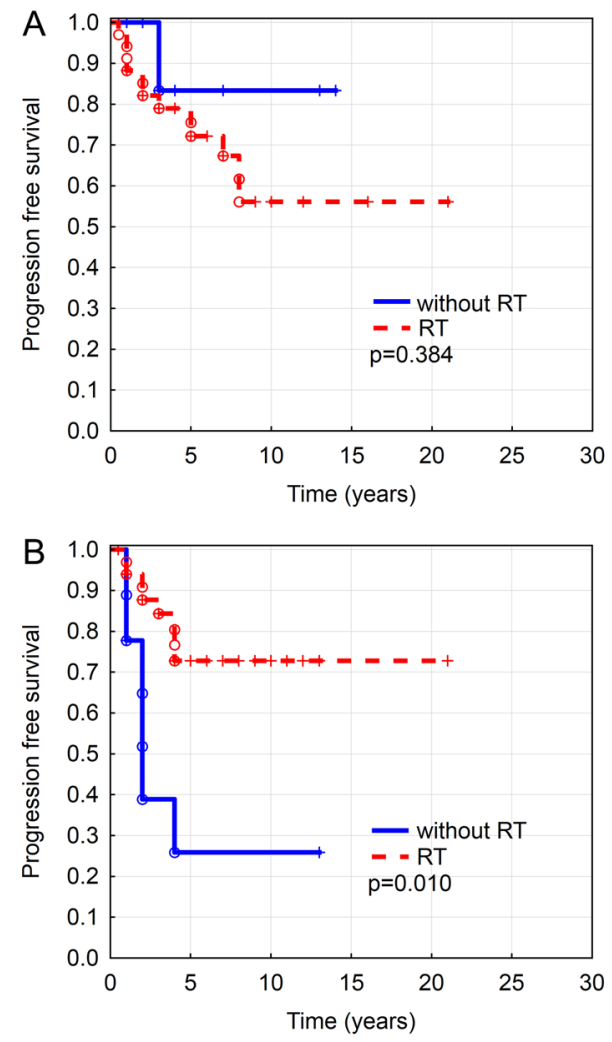

Figure 2

The differences in progression-free survival between patients at high risk of local recurrence (positive margins add/or extrathyroidal extension) with (the red line) and without (the blue line) adjuvant radiotherapy following initial surgical procedure. (A) MTC recurrence in thyroid bed. (B) MTC recurrence in lymph nodes. disease received radiotherapy previously. The differences between hereditary and sporadic subgroups were not significant considering the number of recurrences and response to radiotherapy.

Considering the impact of radiation doses on treatment outcomes, a lower frequency of MTC relapses (47\%) was observed in a subgroup who received the doses of $60 \mathrm{~Gy}$ and higher compared to a subgroup treated with lower doses. However, the differences were not significant (Table 3).

The mean time between the last surgery before radiotherapy and the diagnosis of local recurrence in the whole group was 4.5 years (range $0.5-22$ years).
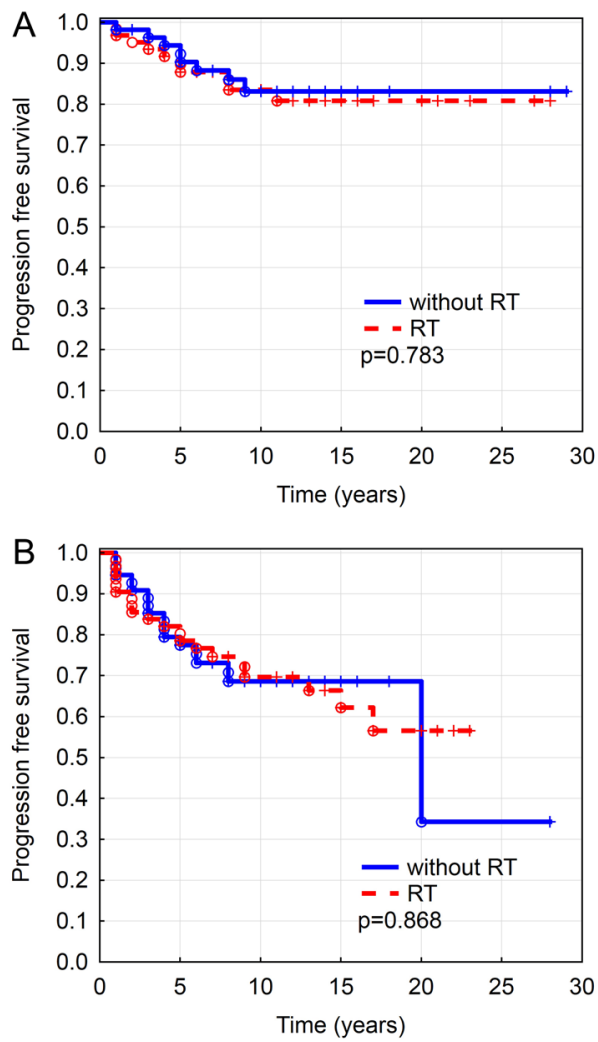

Figure 3

The differences in progression-free survival between $\mathrm{N} 1$ patients with (the red line) and without (the blue line) adjuvant radiotherapy following primary surgical procedure. (A) MTC recurrence in thyroid bed. (B) MTC recurrence in lymph nodes.

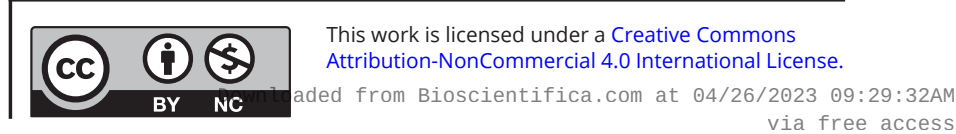



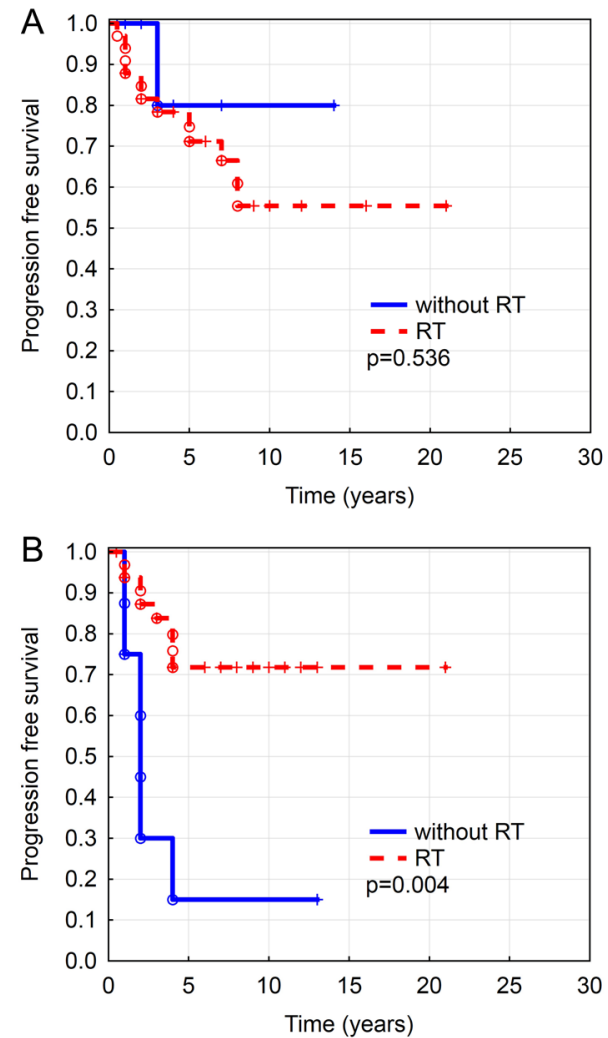

Figure 4

The differences in progression-free survival between N1 patients at high risk of local recurrence (positive margins add/or extrathyroidal extension) with (the red line) and without (the blue line) adjuvant radiotherapy following primary surgical procedure. (A) MTC recurrence in thyroid bed. (B. MTC recurrence in lymph nodes.

During the first five years of follow-up, 78 recurrences were diagnosed, among them $74 \%$ of relapses occurred in patients after adjuvant radiotherapy and $70 \%$ of relapses occurred in patients without radiotherapy.

Male sex, high local MTC stage, N1 feature and previous radiotherapy were significant factors related to a higher risk of local MTC recurrence in univariate analyses. However, in multivariate analysis, only the N1 feature remained statistically significant (Table 4).

Table 3 Radiation doses (adjuvant radiotherapy) and the percentage of medullary thyroid cancer (MTC) relapses.

\begin{tabular}{|c|c|}
\hline $\begin{array}{l}\text { Radiation } \\
\text { dose (Gy) }\end{array}$ & $\begin{array}{l}\text { A whole subgroup } \\
\text { subjected to adjuvant } \\
\text { radiotherapy }\end{array}$ \\
\hline $40-50$ & 7 \\
\hline 50-60 & 35 \\
\hline $60-70$ & 71 \\
\hline$>70$ & 2 \\
\hline
\end{tabular}

\begin{tabular}{ccc}
$\begin{array}{c}\text { MTC } \\
\text { recurrence }\end{array}$ & & $\begin{array}{c}\text { No } \\
\text { recurrence }\end{array}$ \\
\cline { 1 - 1 } $\begin{array}{c}\text { 6(86\%) } \\
21(60 \%)\end{array}$ & & 1 \\
$33(45 \%)$ & & 38 \\
1 & & 1 \\
\hline
\end{tabular}

\section{Discussion}

In the present study, we did not demonstrate a beneficial impact of postsurgical adjuvant radiotherapy on the risk of local relapse in MTC patients. In the whole group, no statistically significant reduction in the number of recurrences in patients after radiotherapy in comparison to patients without radiotherapy was noticed. We did not observe any differences between patients with hereditary and sporadic disease.

The published data are contradicting as it is difficult to confirm or refute the benefit of radiotherapy in a noncontrolled observational cohort studies. Terazakis et al. evaluated the influence of radiotherapy on the course of non-anaplastic thyroid cancer (including 12 MTC patients) and it did not show any beneficial effect on the local MTC control and survival (19). While, in 1996 Brierley et al. achieved a better local control only in irradiated, high-risk patients with extrathyroidal extension, positive surgical margins or lymph node metastases, in whom radiotherapy improved 10-year recurrence-free survival from 52 to $86 \%$. Nevertheless, Brierley et al. did not observe any beneficial impact of postoperative radiotherapy in the whole analyzed group of 46 MTC patients who received radiotherapy at doses ranged from 20 to 75 Gy (12). However, the other authors demonstrated a better local control related to radiotherapy, particularly in a group of patients with advanced disease. In 1985 Tubiana et al. observed a longterm effect in patients who underwent radiotherapy due to inoperable MTC (11). In 1996 Fife et al. analyzed a group of 51 patients and concluded that radiotherapy could be used to improve local control in patients after non-radical surgical approach and recommended high radiation doses, at least $60 \mathrm{~Gy}$ (20). Similar results, supporting radiotherapy in MTC, were published in 2016 (14). Compagnon et al. proposed to apply adjuvant radiotherapy after surgery in high-risk patients as a protective method providing a good local control (14). The authors of the most recent analysis, which involved a group of 302 MTC patients, among whom 51 patients after EBRT, suggested that EBRT should be considered in patients with positive surgical margins as it may improve local control rates (21).

In our study, we observed a small group of high-risk MTC patients after non-radical surgery. Probably due to a small number of analyzed patients as well as due to the lack of complete data regarding surgical treatment resulting from a retrospective nature of our study, we were not able to prove radiotherapy benefits in terms of thyroid bed recurrences in the group, in which radiotherapy is usually recommended. However, we demonstrated a beneficial

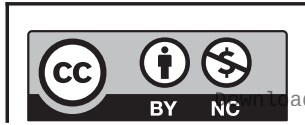

This work is licensed under a Creative Commons Attribution-NonCommercial 4.0 International License. ded from Bioscientifica.com at 04/26/2023 09:29:32AM via free access 
Table 4 The results for univariate and multivariate analyses for PFS.

\begin{tabular}{|c|c|c|c|c|c|}
\hline \multirow[b]{2}{*}{ Factor } & & \multicolumn{2}{|c|}{ Univariate analyses } & \multicolumn{2}{|c|}{ Multivariate analysis } \\
\hline & & OR & $P$ & OR & $P$ \\
\hline \multirow[t]{2}{*}{ Sex } & $\mathrm{F}$ & 1.0 (ref.) & & 1 & \\
\hline & $\mathrm{M}$ & 1.86 & 0.022 & 1.56 & 0.134 \\
\hline \multirow[t]{2}{*}{ RET germline mutation } & Present & 1 & & 1 & \\
\hline & Absent & 0.63 & 0.108 & 0.76 & 0.398 \\
\hline \multirow[t]{3}{*}{ T feature } & $\mathrm{T} 1-2$ & 1.0 & & 1 & \\
\hline & $\mathrm{T} 3-4$ & 2.39 & 0.003 & 1.53 & 0.181 \\
\hline & $\mathrm{Tx}$ & 2.40 & 0.014 & 2.27 & 0.039 \\
\hline \multirow[t]{3}{*}{$\mathrm{N}$ feature } & NO & 1.0 & & 1 & \\
\hline & N1 & 14.11 & 0.0001 & 10.88 & 0.0001 \\
\hline & $\mathrm{Nx}$ & 3.62 & 0.090 & 2.61 & 0.222 \\
\hline \multirow[t]{2}{*}{ Radiotherapy } & No RT & 1.0 & & 1 & \\
\hline & RT & 2.02 & 0.007 & 0.92 & 0.782 \\
\hline
\end{tabular}

effect of postoperative radiotherapy on the risk of lymph node recurrences in a high-risk group. This result seems to be even more important as the necessity of elective radiotherapy in MTC is still discussed. Resignation from elective radiotherapy may allow to reduce the radiation field and in consequence to reduce treatment-related complications. Nevertheless, the significant effect of adjuvant radiotherapy on the risk of nodal relapses supports the idea of elective radiotherapy in MTC patients with a high local stage or after non-radical surgical approach.

We did not observe a significant reduction in the number of subsequent recurrences in patients in whom radiotherapy followed surgical resection of MTC relapse. More than $50 \%$ of these patients were diagnosed due to another MTC recurrence, mainly in thyroid bed.

The study group was heterogeneous regarding radiotherapy techniques, which differed during a longtime period we observed our patients. MTC is characterized by a natural long-term course and long-term survival. MTC recurrences may occur many years after surgery (even 20 years in our material), thus radiotherapy did not show any impact on the time between surgeries and relapse diagnosis. On the other hand, long-term follow-up constitutes an important advantage of our report.

Currently, when we have new promising treatment options for advanced MTC, thanks to approval of multikinase inhibitors (22), the decision about radiotherapy has to be cautious due to its relatively low efficacy and treatment-related side effects.

The lack of precise data on radiotherapy-related side effect is an important limitation of our study. The data we had did not allow for the evaluation of radiotherapy complications according to any point scale, particularly regarding the patients treated in earlier years. Numerous patients complained of skin reaction, tracheitis, and esophagitis. Tracheostomy or PEG placement was not necessary in any patient. There were no severe late complications (including spinal cord damage).

As it was mentioned earlier, our group was relatively large with a long-term follow-up, but heterogeneous, because radiotherapy was applied at different times with the use of different techniques. The lack of homogenous group did not allow in general to call in doubt the use of radiotherapy in MTC patients, particularly in high-risk subjects with accepted indications for treatment. Only randomized, prospective clinical trial could unequivocally resolve the question related to the use of adjuvant radiotherapy in MTC. However, such study seems to be too difficult due to the rarity of MTC. The probability of conducting a prospective trial in the nearest feature is low. Thereby, we should base on our knowledge and retrospective data.

We would rather like to pay attention to the limitation of radiotherapy as a method of MTC treatment than to confirm its beneficial role in the achievement of local disease control and to emphasize the necessity of treatment personalization in particular, when elevated serum calcitonin level may be the first signal of MTC dissemination in the further disease course and the need of systemic therapy in the future. Our data do not demonstrate that radiotherapy improves local control in MTC and suggest low MTC radiosensitivity and doubtful efficacy, especially in low-risk patients. Radiotherapy may be considered only in selected cases, in patients after non-radical surgery, with positive margins or neoplastic infiltration of adjacent tissues. The current ATA guidelines recommend radiotherapy only in a case of a very high risk of local relapse (6). Our study, based on a relatively high number of MTC patients and an adequate - considering its biology - time of follow-up, confirmed a strategy already applied. The results of our analysis may help the clinicians to resolve difficult questions in real life. 


\section{Conclusion}

Adjuvant radiotherapy has a limited importance in the treatment of MTC. It should be considered for high-risk patients with local relapse. The presence of RET mutation does not influence the response to radiation.

\section{Declaration of interest}

The authors declare that there is no conflict of interest that could be perceived as prejudicing the impartiality of the research reported.

\section{Funding}

The project was supported by the Polish National Center of Research and Development MILESTONE project - Molecular diagnostics and imaging in individualized therapy for breast, thyroid and prostate cancer, grant no. STRATEGMED 2/267398/4/NCBR/2015.

\section{References}

1 Pacini F, Castagna MG, Cipri C \& Schlumberger M. Medullary thyroid carcinoma. Clinical Oncology 201022 475-485. (https://doi. org/10.1016/j.clon.2010.05.002)

2 Maxwell JE, Sherman SK, O'Dorisio TM \& Howe JR. Medical management of metastatic medullary thyroid cancer. Cancer 2014 120 3287-3301. (https://doi.org/10.1002/cncr.28858)

3 Leboulleux S, Baudin E, Travagli J-P \& Schlumberger M. Medullary thyroid carcinoma. Clinical Endocrinology 200461 299-310.

4 Kloos RT, Eng C, Evans DB, Francis GL, Gagel RF, Gharib H, Moley JF, Pacini F, Ringel MD, Schlumberger M, et al. Medullary thyroid cancer: management guidelines of the American Thyroid Association. Thyroid 200919 565-612. (https://doi.org/10.1089/ thy.2008.0403)

5 Moers AM, Landsvater RM, Schaap C, Jansen-Schillhorn van Veen JM, de Valk IA, Blijham GH, Höppener JW, Vroom TM, van Amstel HK \& Lips CJ. Familial medullary thyroid carcinoma: not a distinct entity? Genotype-phenotype correlation in a large family. American Journal of Medicine 1996101 635-641. (https://doi. org/10.1016/s0002-9343(96)00330-0) Machens A, Moley JF, Pacini F, et al. Revised American Thyroid Association guidelines for the management of medullary thyroid carcinoma. Thyroid 201525 567-610. (https://doi.org/10.1089/ thy.2014.0335)

7 Schlumberger M, Bastholt L, Dralle H, Jarzab B, Pacini F, \& Smit JWA. 2012 European Thyroid Association guidelines for metastatic medullary thyroid cancer. European Thyroid Journal 2012 1 5-14. (https://doi.org/10.1159/000336977)

8 Steinfeld AD. The role of radiation therapy in medullary carcinoma of the thyroid. Radiology 1977123 745-746. (https://doi. org/10.1148/123.3.745)

9 Samaan NA, Schultz PN \& Hickey RC. Medullary thyroid carcinoma: prognosis of familial versus sporadic disease and the role of radiotherapy. Journal of Clinical Endocrinology and Metabolism 198867 801-805. (https://doi.org/10.1210/jcem-67-4-801)
6 Wells SA, Asa SL, Dralle H, Elisei R, Evans DB, Gagel RF, Lee N,

10 Martinez SR, Beal SH, Chen A, Chen SL \& Schneider PD. Adjuvant external beam radiation for medullary thyroid carcinoma. Journal of Surgical Oncology 2010102 175-178. (https://doi.org/10.1002/ jso.21557)

11 Tubiana M, Haddad E, Schlumberger M, Hill C, Rougier P \& Sarrazin D. External radiotherapy in thyroid cancers. Cancer 1985 55 (9 Supplement) 2062-2071. (https://doi.org/10.1002/10970142(19850501)55:9+<2062::aid-cncr2820551406>3.0.co;2-o)

12 Brierley J, Tsang R, Simpson WJ, Gospodarowicz M, Sutcliffe S \& Panzarella T. Medullary thyroid cancer: analyses of survival and prognostic factors and the role of radiation therapy in local control. Thyroid 19966 305-310. (https://doi.org/10.1089/thy.1996.6.305)

13 Schwartz DL, Rana V, Shaw S, Yazbeck C, Ang KK, Morrison WH, Rosenthal DI, Hoff A, Evans DB, Clayman GL, et al. Postoperative radiotherapy for advanced medullary thyroid cancer - local disease control in the modern era. Head and Neck 200830 883-888. (https:// doi.org/10.1002/hed.20791)

14 Compagnon F, Zerdoud S, Rives M, Laprie A, Sarini J, Grunenwald S, Chaltiel L \& Graff P. Postoperative external beam radiotherapy for medullary thyroid carcinoma with high risk of locoregional relapse. Cancer Radiotherapie 201620 362-369. (https://doi.org/10.1016/j. canrad.2016.05.010)

15 Terezakis SA \& Lee NY. The role of radiation therapy in the treatment of medullary thyroid cancer. Journal of the National Comprehensive Cancer Network 20108 532-540; quiz 541. (https://doi.org/10.6004/ jnccn.2010.0041)

16 Brierley JD. Update on external beam radiation therapy in thyroid cancer. Journal of Clinical Endocrinology and Metabolism 201196 2289-2295. (https://doi.org/10.1210/jc.2011-1109)

17 Nutting CM, Convery DJ, Cosgrove VP, Rowbottom C, Vini L, Harmer C, Dearnaley DP \& Webb S. Improvements in target coverage and reduced spinal cord irradiation using intensity-modulated radiotherapy (IMRT) in patients with carcinoma of the thyroid gland. Radiotherapy and Oncology 200160 173-180. (https://doi. org/10.1016/s0167-8140(01)00382-6)

18 Bhide SA, Newbold KL, Harrington KJ \& Nutting CM. Clinical evaluation of intensity-modulated radiotherapy for head and neck cancers. British Journal of Radiology 201285 487-494. (https://doi. org/10.1259/bjr/85942136)

19 Terezakis SA, Lee KS, Ghossein RA, Rivera M, Tuttle RM, Wolden SL, Zelefsky MJ, Wong RJ, Patel SG, Pfister DG, et al. Role of external beam radiotherapy in patients with advanced or recurrent nonanaplastic thyroid cancer: Memorial Sloan-Kettering Cancer Center experience. International Journal of Radiation Oncology, Biology, Physics 200973 795-801. (https://doi.org/10.1016/j. ijrobp.2008.05.012)

20 Ronga G, Filesi M, Ventroni G, Vestri AR \& Signore A. Value of the first serum thyroglobulin level after total thyroidectomy for the diagnosis of metastases from differentiated thyroid carcinoma. European Journal of Nuclear Medicine 199926 1448-1452. (https://doi. org/10.1007/s002590050477)

21 Groen A, Beckham T, Links T, Sherman E, Tuttle RM, Fagin J, Sasha A, Sabol C, Tsai CJ, McBride S, et al. Impact of surgery and external beam radiation therapy on local control in the treatment of medullary thyroid carcinoma. International Journal of Radiation Oncology, Biology, Physics 2018102 e230. (https://doi.org/10.1016/j. ijrobp.2018.07.783)

22 Krajewska J, Gawlik T \& Jarzab B. Advances in small molecule therapy for treating metastatic thyroid cancer. Expert Opinion on Pharmacotherapy 201718 1049-1060. (https://doi.org/10.1080/14656 566.2017.1340939)

Received in final form 7 November 2019

Accepted 28 November 2019

Accepted Manuscript published online 28 November 2019
This work is licensed under a Creative Commons Attribution-NonCommercial 4.0 International License. ded from Bioscientifica com at 04/26/2023 09:29:32AM 